\title{
Incremental Provision of QoS Discarding Non-feasible End-to-End Paths ${ }^{\star}$
}

\author{
Alfonso Gazo-Cervero, José Luis González-Sánchez, \\ and Francisco J. Rodríguez-Pérez \\ Computer Science Department, University of Extremadura, \\ Escuela Politécnica de Cáceres, Av/ Universidad s/n, 10071 - Cáceres, Spain \\ \{agazo, jlgs, fjrodri\}@unex.es
}

\begin{abstract}
Trying to find a practical solution for QoS provision capabilities over IP networks has been subject of a great research effort during last years. The main aim of the proposal presented in this paper is to allow QoS provision over IP networks without the requirement of upgrading every router of the Internet. Upgrading some routers within the network is still needed, but no constraints do exist related to which routers might be upgraded.
\end{abstract}

\section{Introduction and Related Work}

Enhancing the current Internet to include QoS provision capabilities is still an open issue that has been researched since the Integrated Services (IntServ) specification. One of the approaches is to enhance every router in the Internet so that QoS flows are prioritized at each hop in case of congestion. Another approach is to apply strict admission control mechanisms to every incoming traffic flow, be a QoS flow or not, so congestion would be avoided. Finally, the overprovisioning approach tries to assure that there are enough resources in the network to satisfy traffic demand.

Bandwidth is an important issue for routers which are not in the backbone, so the overprovisioning approach does not seem appropriate for them. For the other approaches to be applied, current equipment must be modified. However, current size of the Internet indeed makes its modification a challenging task. Because of this, our work describes a generalized proposal that allows QoS provision to be deployed over current protocols in the Internet. Modifications to current equipment are still needed, but it is not mandatory to deploy them at every node within the Internet. We have called it incremental, because it allows providers to avoid migrating their whole network, but only parts of it. Our proposal is designed to work over a variety of different network technologies to achieve QoS provision such as MultiProtocol Label Switching (MPLS) and Open Shortest Path First (OSPF). It uses an automated manager that is inspired on the Bandwidth Broker (BB) described in the two-bit Differentiated Services (DiffServ) architecture.

Automated management is still an open issue in QoS provision. It has been researched in many works, some of them suggesting a centralized manager [12] or distributed management [3] 4 .

\footnotetext{
* This work is sponsored in part by the Regional Government of Extremadura, Spain (Education,

Science and Technology Council) under GRANT N ${ }^{\mathrm{O}}$ PRIA06145.
} 


\section{Overlay QoS Framework}

Our proposal is based on the creation and management of an overlay network. The overlay network is managed by an agent within the Autonomous System (AS), called Network Broker (NB). A number of edge nodes within the network include signaling capabilities to communicate to the NB. Users that are willing to request any guarantees for their traffic also include signaling capabilities. The proposal described in this paper extends the functions suggested for the BB of the DiffServ architecture. New functions incorporated include overlay network management and Traffic Engineering (TE) functions, if available. As a result, the element that carry out all these tasks is called NB. More details on this can be found in [5].

\subsection{Overlay Network}

Several Internet overlays have been designed in the past for various purposes, but what makes a difference in an overlay network for QoS provision is that there is a need for end-to-end guarantees. Our overlay network (figure 1) is defined as follows: let $G(V, E)$ describe an AS network, where $V$ is the set of nodes and $E$ the set of links. Overlay network is described by $G^{\prime}\left(V^{\prime} \subseteq V, E^{\prime} \subseteq E\right)$ if $\forall v \in V: v \in V^{\prime} \Leftrightarrow i s_{\text {_qnode }}(v)$. The is-qnode function is defined as follows: if node is an edge router, is-qnode returns true if and only if node has classifying, marking, shaping, policing, scheduling and NB signaling capabilities; and if node is a core router, is-qnode returns true if and only if node has scheduling and policing capabilities.

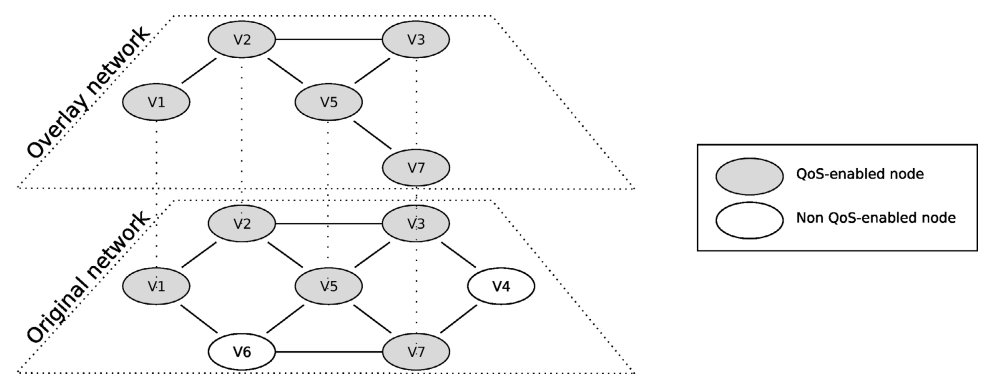

Fig. 1. Original and overlay network representation

\subsection{Resource Management}

The NB manages a database that contains assigned bandwidth to every traffic class at every link in the AS. Once admission control phase if passed, this database is updated to reflect the new bandwidth assignments at all links along calculated path. If we consider $T=\left\{t_{0}, \ldots, t_{n}\right\}$ the set of different traffic classes excluding best effort, let $C(e)$ be link capacity for link $e \in E^{\prime}, R_{t}(e)$ be the residual bandwidth remaining for traffic class $t$ at link $e \in E^{\prime}$, the maximum bandwidth assignment $B_{t}(e)$ for traffic class $t$ at link $e \in E^{\prime}$ is limited by $C(e) \cdot \lambda_{t}$. The $\lambda_{t}$ factor is bounded by $0<\lambda_{t}<1$ and represents 
the proportional amount of bandwidth that would be available for traffic class $t$ at every link. In addition, each $\lambda_{t}$ must be chosen so that the following expression is fulfilled:

$$
0<\sum_{\forall t \in T} \lambda_{t}<1
$$

Residual bandwidth $R_{B E}(e)$ for best effort traffic class at link $e \in E$ is bounded by the following expression:

$$
\forall e \in E: \begin{cases}C(e) \cdot\left(1-\sum_{\forall t \in T} \lambda_{t}\right) \leq R_{B E}(e) \leq C(e) & \text { if } e \in E^{\prime} \\ R_{B E}(e)=C(e) & \text { if } e \notin E^{\prime}\end{cases}
$$

\subsection{Routing}

The NB has two modes of operation. The mode of operation is selected depending on the characteristics of the Interior Gateway Protocol (IGP) used at the AS. If explicit routing is allowed, NB builds explicit routes based on its own routing information for Quality of Service (QoS) flows. This is what we call Routing Mode, and explicit routes are signaled to q-routers. But if explicit routing is not available, the NB pre-calculate paths that the routing protocol is supposed to establish. This calculation is done at the admission control phase. Unlike the Routing Mode case, if explicit routing is not available, the NB calculate paths to accomplish admission control and resource management at the NB. Neither routers nor $q$-routers are signaled, because signaling them would imply a modification of the routing protocol, which we try to avoid.

\section{Simulation Results}

To test the behavior of our proposal, the Network Simulator (ns-2) has been enhanced to incorporate the architecture described. The following simulation results are obtained using a representation of the AT\&T topology publicly available. The distribution of $q$ nodes within the topology is driven by a q-prob parameter, which represents the probability of a node to behave as a $q$-node and hence to take part on the QoS overlay topology.

Either Routing and Non-Routing modes have been implemented in the simulator. Figure 2a demonstrates the behavior of the NB in Routing Mode for a number of scenarios with different values for the q-prob parameter and a variable number of flows. Figure $2 \mathrm{~b}$ demonstrates the behavior of the NB in Non Routing Mode under the same scenarios and traffic load as the previous figure. The percentage of requested blocked flows increases when traffic load is also increased, as expected. When Non Routing Mode is used, TE techniques could not be applied thus causing a faster increase in the number of blocked flows compared to the case in which Routing Mode is used.

Considering the $q$-prob parameter, the above figures show that, for the same scenario and traffic load, the more the number of $q$-nodes that are present within the network, the less the percentage of blocked flows. 


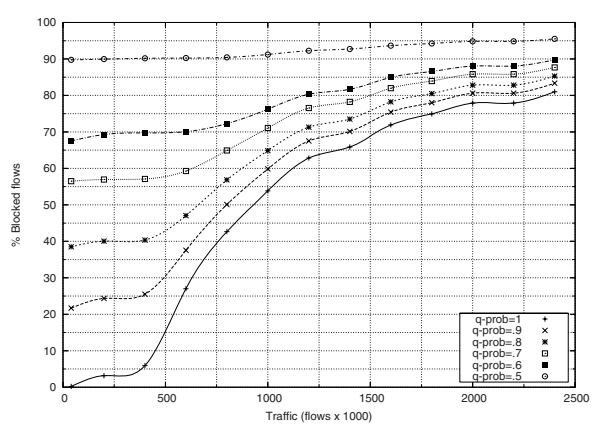

(a) Using the WSGP routing algorithm

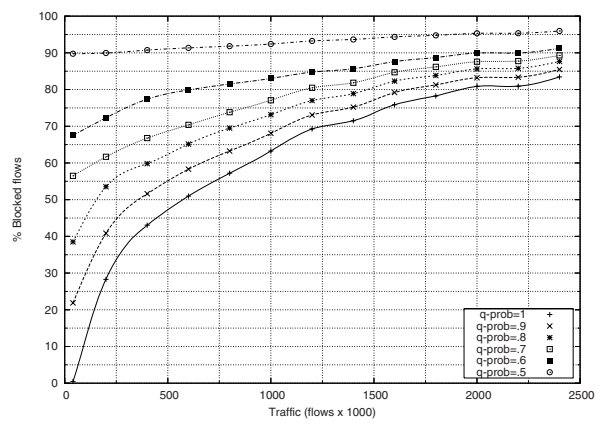

(b) Using the SGP routing algorithm

Fig. 2. Percentage of blocked flows over the AT\&T topology

\section{Conclusions and Future Work}

Main novelty of this proposal is the possibility that not every node within an AS are needed to support QoS provision mechanisms, but only some of them. Other proposals already exist with this approach, but they often require complex protocols that also interfere with best-effort service provision or have constraints related to which nodes must support QoS provision mechanisms.

Results section of this paper presents how decreasing the amount of nodes which include QoS provision mechanisms has an impact over the QoS provision global capacity at the AS. But it is also shown that QoS provision is still possible under some circumstances. We have focused on MPLS and OSPF network technologies, yet its generalized approach let this proposal to be deployed over a wider range of technologies.

\section{References}

1. Scoglio, C., Anjali, T., Oliveira, J.C., Akyildiz, I.F., Uhl, G.: TEAM: A Traffic Engineering Automated Manager for DiffServ-based MPLS Networks. IEEE Communications Magazine 42 (2004) 134-145

2. Zhang, Z., Duan, Z., Hou, Y.T.: On scalable network resource management using Bandwidth Brokers. In: IEEE Network Operations and Management Symposium. (2002)

3. Bhatnagar, S., Nath, B.: Distributed Admission Control to Support Guaranteed Services in Core-Stateless Networks. In: IEEE INFOCOM 2003. Volume 3. (2003) 1659-1669

4. Lima, S., Carvalho, P., Freitas, V.: Distributed Admission Control for QoS and SLS Management. Journal of Network and Systems Management. Special issue "Distributed Management of Networks and Services" 12 (2004) 397-426

5. Gazo-Cervero, A., González-Sánchez, J.L.: Incremental QoS deployment based on Network Brokers. In: HET-NETs'04. 2nd International Working Conference. Performance Modelling and Evaluation of Heterogeneous Networks. (2004) 